\title{
TERT alleviates irradiation-induced late rectal injury by reducing hypoxia-induced ROS levels through the activation of NF-kB and autophagy
}

\author{
QI LIU ${ }^{1,3}$, YONG SUN ${ }^{1,3}$, YUEFENG LV ${ }^{4}$, ZIYU LE ${ }^{2,3}$, YUHU XIN ${ }^{1,3}$, PING ZHANG ${ }^{1,3}$ and YONG LIU ${ }^{1-3}$ \\ ${ }^{1}$ Cancer Research Institute and ${ }^{2}$ Department of Radiation Oncology, Fudan University Shanghai Cancer Center; \\ ${ }^{3}$ Department of Oncology, Shanghai Medical College, Fudan University, Shanghai 200032; ${ }^{4}$ Department of Obstetrics, \\ Shiyan Taihe Hospital, Hubei University of Medcine, Shiyan, Hubei 442000, P.R. China
}

Received November 19, 2015; Accepted June 28, 2016

DOI: $10.3892 /$ ijmm.2016.2673

\begin{abstract}
The hypoxic microenvironment which is present following irradiation has been proven to promote radiationinduced injury to normal tissues. Previous studies have demonstrated that telomerase reverse transcriptase (TERT) is regulated by hypoxia, and that it plays a protective role in the process of wound repair. However, its effects on radiationinduced injury remain unclear. In this study, we examined the effects of human TERT on irradiation-induced late rectal injury in fibroblasts under hypoxic conditions. We also performed in vivo experiments. The rectums of 5-week-old female C57BL/6N mice were irradiated locally with a single dose of $25 \mathrm{~Gy}$. We then examined the fibrotic changes using hematoxylin and eosin staining, and Masson's staining. The expression of hypoxia inducible factor- $1 \alpha$ (HIF-1 $\alpha)$ and TERT was analyzed by immunohistochemistry. In in vitro experiments, apoptosis, reactive oxygen species (ROS) production and the autophagy level induced by exposure to hypoxia were assayed in fibroblasts. The association between TERT, nuclear factor- $\kappa \mathrm{B}(\mathrm{NF}-\kappa \mathrm{B})$ and the autophagy level was examined by western blot analysis. The antioxidant effects of TERT were examined on the basis of the ratio of glutathione to glutathione disulfide (GSH/GSSG) and mitochondrial membrane potential. Rectal fibrosis was induced significantly at 12 weeks following irradiation. The HIF-1 $\alpha$ and TERT expression levels increased in the fibrotic region. The TERT-overexpressing fibroblasts (transfected with an hTERT-expressing lentiviral vector) exhibited reduced apoptosis, reduced ROS production, a higher autophagy level, a higher GSH/GSSG ratio and stable mitochondrial membrane potential compared with the fibro-
\end{abstract}

Correspondence to: Professor Yong Liu, Cancer Research Institute, Fudan University Shanghai Cancer Center, 270 Dong An Road, Shanghai 200032, P.R. China

E-mail: drliuyong@hotmail.com

Key words: radiation, late rectal injury, hypoxia, telomerase reverse transcriptase, autophagy blasts in which TERT had been silenced by siRNA. NF- $\kappa$ B was activated by TERT, and the inhibition of TERT reduced the autophagy level in the fibroblasts. These results demonstrate that TERT decreases cellular ROS production, while maintaining mitochondrial function and protecting the cells from hypoxia-induced apoptosis, which may thus attenuate the effects of irradiation-induced hypoxia on rectal injury following irradiation.

\section{Introduction}

Radiation therapy is widely utilized for the treatment of pelvic tumors. The rectum is a high-risk organ for radiation-induced injury, which is a dose-limiting factor of pelvic radiotherapy. Chronic damage characterized by connective tissue growth, tissue remodeling and fibrosis impair rectal function and worsen the quality of life of cancer patients (1).

Previous studies have demonstrated that radiation can induce hypoxia via vessel damage and tissue remodeling, which then promote radiation-induced injury to the lungs, brain and rectum (2-4). Further studies have indicated that hypoxic conditions exist in normal rectal tissues following irradiation. Hypoxia increases hypoxia inducible factor- $1 \alpha$ (HIF- $1 \alpha$ ) and vascular endothelial growth factor (VEGF) expression, which aggravates inflammation and injury $(4,5)$. Furthermore, hypoxia can stabilize HIF-1 $\alpha$ expression and stimulate the transforming growth factor (TGF)- $\beta$-dependent synthesis of collagen and extracellular matrix (ECM) proteins (6). Hypoxia is also an inducer of reactive oxygen species (ROS) and increased leukocyte migration and vascular permeability, and it upregulates TGF- $\beta$ and promotes collagen formation, which are of vital importance in the development of fibrosis $(7,8)$. In addition, ROS exert deleterious effects on neurological organs through damage to cellular macromolecules and membranes, which is associated with low levels of antioxidants, high concentrations of unsaturated fatty acids and the availability of redox-active iron (9).

Telomeres are specialized structures that protect chromosomal ends from degradation, end fusion and rearrangement, and preserve chromosomal integrity and stability (10). Telomerase consists of telomerase reverse transcriptase (TERT), 
telomerase-associated proteins, and the telomerase RNA (TR) component (TERC) (11). The catalytic subunit of the telomerase holoenzyme, TERT, which is essential to the maintenance of telomerase, contributes to cell proliferation $(12,13)$, preventing cellular senescence $(14,15)$, and promoting the repair of damaged tissue and wound healing $(16,17)$. Hypoxic conditions induce a DNA damage response by causing telomere damage. In response to this damage, HIF-1 $\alpha$ induces telomerase in order to heal the damaged chromosome ends (18). TERT also undergoes subcellular localization to the mitochondria in neurons upon cellular stress in Alzheimer's disease (AD) and protects against brain injury (9). Furthermore, TERT reduces intracellular ROS production and inhibits ROS-mediated apoptosis $(6,19)$.

However, the role of TERT in radiation-induced injury to normal tissue and the associatoin between TERT and hypoxia remain unclear. In this study, we examined TERT expression in rectal tissue following irradiation and examined its effects on hypoxia-induced fibroblast apoptosis.

\section{Materials and methods}

Cell culture and animals. Human skin fibroblasts (FBs) were purchased from the Cell Bank of the Chinese Academy of Sciences (Shanghai, China). Both the FBs and human TERT (hTERT)-overexpressing fibroblasts (FB-TERT fibroblasts) that were used in this study were not used for more than 3 months and/or 20 passages. The cells were cultured in Dulbecco's modified Eagle's medium (DMEM; Biowest, Nuaillé, France), supplemented with $1 \%$ penicillin/streptomycin and $10 \%$ fetal bovine serum (FBS; Biowest) in free gas exchange with the atmospheric air at $37^{\circ} \mathrm{C}$. Exposure to hypoxia (for 24 or $48 \mathrm{~h}$ ) was carried out using a tri-gas incubator $\left(37^{\circ} \mathrm{C}, 5 \% \mathrm{CO}_{2}, 93 \% \mathrm{~N}_{2}, 2 \% \mathrm{O}_{2}\right.$; YCP-50S; Changsha Huaxi Electronic Technology Co., Ltd., Hunan, China). We also used the autophagy inhibitor, 3-methyladenine (3-MA; Selleckchem, Houston, TX, USA), at the concentration of $5 \mathrm{mM}$, the ROS scavenger, N-acetylcysteine (NAC; Sigma, St. Louis, CA, USA) at the concentration of $3 \mathrm{mM}$, the p-NF- $\kappa$ B inhibitor, BAY 11-7082 (Selleckchem) at the concentration of $10 \mu \mathrm{M}$ and DMSO (Sigma) at the concentration of $10 \mu \mathrm{M}$. Thirty female C57BL/6N mice (age, 5 weeks; weight, 15-25 g) were purchased from Shanghai Sippr/BK Laboratory Animals Co., Ltd. (Shanghai, China). Prior to irradiation, the mice were housed in a pathogen-free room under controlled temperature and humidity for 1 week. Following irradiation, all mice were divided into 6 groups (the control group, and the post-RT group for 1, 7, 14, 28 and 90 days).

$X$-ray irradiation. X-ray irradiation (RT) was performed with a single dose of $25 \mathrm{~Gy}$ and at a dose rate of $3.69 \mathrm{~Gy} / \mathrm{min}$ using the Small Animal Radiation Research Platform (SARRP; Baltimore, MD, USA). The mice were anesthetized by pentobarbitol sodium (60-80 $\mu \mathrm{l})$ and were then irradiation was applied locally to the rectums (10 $\mathrm{mm}$ in diameter). Following irradiation, the mice were housed in cages and supplied with a standard laboratory diet and water. The study was performed in strict accordance with the recommendations in the Guide for the Care and Use of Laboratory Animals of Fudan University. The protocol was approved by the Committee on the Ethics of Animal Experiments of Fudan University.
Lentiviral vector production. For construction of the hTERT-expressing lentiviral vector, a cDNA-coding hTERT (NF-198253.2, 3399-bp cDNA) was synthesized and cloned into the Bam HI and XbaI restriction endonuclease sites of the pLenO-DCE vector (Invabio Biotechnology Ltd., Shanghai, China). The pLenO-DCE-hTERT plasmid was then acquired, which also encodes green fluorescent protein (GFP). The products were transformed into competent fibroblasts. The emerged cell colonies were cultured, plasmid DNA was isolated, digested and sequenced to confirm that the correct clone had been made. After the correct sequence was confirmed, lentiviral vector particles were produced in accordance with the manufacturer's instructions (Invitrogen, Carlsbad, CA, USA). A control vector driving the expression of GFP (pLenO-DCE-GFP) was also generated. pRsv-REV, Pmd1g-Prre, Pmd2g and pLenO-DCEhTERT were co-transfected into 293T cells (American Type Culture Collection; ATCC, Manassas, VA, USA) and viral supernatants were harvested 48 and $72 \mathrm{~h}$ post-transfection and concentrated using 4 rounds of ultracentrifugation. The cell surpernatant, which contained viral particles, was collected and stored at $-80^{\circ} \mathrm{C}$. Viral titers were detected by the double dilution method on $293 \mathrm{~T}$ cells.

Tissue sampling, histological analysis and immunohistochemistry. The mice were sacrificed by cervical dislocation. Rectal tissue samples were collected on days $1,7,14,28$, and 90 postirradiation. The sections were fixed using $4 \%$ paraformaldehyde and then embedded in paraffin. The sections were then cut to $4 \mu \mathrm{m}$ thickness for hematoxylin and eosin (H\&E) staining, Masson's staining and immunohistochemical analysis. For the analysis of the severity of irradiation-induced injury and fibrosis, the levels of mucosal depletion and collagen deposition were analyzed under a microscope (Nikon, Tokyo, Japan). For immunohistochemical analysis, the samples were incubated with anti-HIF-1 $\alpha$ polyclonal antibody (1:150; Medical \& Biological Laboratories Co., Ltd., Nagoya, Japan) or anti-hTERT polyclonal antibody (1:1500; ab183105; Abcam, Cambridge, UK) as the primary antibody overnight at $4^{\circ} \mathrm{C}$. The sections were then incubated with the secondary antibody (1:500; Abcam) and examined under a microscope (Nikon, Tokyo, Japan).

$R N A$ isolation and reverse transcription-quantitative polymerase chain reaction $(R T-q P C R)$. RNA was isolated using a High Pure RNA Isolation kit (RNAprep pure cell/ bacteria kit; Tiangen, Beijing, China) and treated with DNase to eliminate genomic DNA contamination. Total RNA (500 ng) was used as a template for reverse transcription into cDNA using the PrimeScript ${ }^{\mathrm{TM}}$ RT reagent kit (Perfect Real Time; Takara Bio Inc., Shiga, China), which was then used for qPCR. All mRNA expression levels were normalized to $\beta$-actin. The following primers were used for TERT: sense, 5'-CGGAAGA GTGTCTGGAGCAA-3' and antisense, 5'-GGATGAAGCGG AGTCTGGA-3'. qPCR was performed using LightCycler 480 SYBR-Green I Master (Roche Diagnostics, Basel, Switzerland). The $\Delta \Delta \mathrm{Ct}$ method was used for relative quantification.

Western blot analysis. Cells were lysed with RIPA lysis buffer (Roche Diagnostics, Basel, Switzerland), and total protein was quantified using the Pierce BCA Protein Assay kit (Thermo Fisher Scientific, Inc., Waltham, MA, USA). For 
western blot analysis, equal amounts of protein extracts were separated by sodium dodecyl sulfate (SDS)-polyacrylamide gel electrophoresis (PAGE) on a $10 \%(\mathrm{w} / \mathrm{v})$ polyacrylamide gel, followed by electrotransfer onto a BioTrace NC Membrane (Pall Corporation, Pensacola, FL, USA). The blots were blocked for $1 \mathrm{~h}$ with blocking buffer [5\% (w/v) bovine serum albumin, $0.1 \%(\mathrm{v} / \mathrm{v})$ Tween-20 in phosphate-buffered saline (PBS)]. The antibodies used for western blot analysis were as follows: nuclear factor- $\kappa \mathrm{B}(\mathrm{NF}-\kappa \mathrm{B}) / \mathrm{p}-\mathrm{NF}-\kappa \mathrm{B}$ p65 (2884-1; Epitomics, Burlingame, CA, USA), LC3 (M152-3; Medical \& Biological Laboratories Co., Ltd.) and TERT (ab183105; Abcam). This was followed by incubation with horseradish peroxidase-coupled secondary anti-rabbit (Millipore, Billerica, MA, USA) or antimouse antibodies (GeneTex Inc., Irvine, CA, USA). Protein bands were detected using the ECL Blotting Detection Reagents (Thermo Fisher Scientific, Inc.) and imaged and quantified using the Chemioscope Mini system (Bioshine, Shanghai, China). Nuclear-cytoplasmic fractionation was conducted using the NE-PER Nuclear and Cytoplasmic Extraction Reagents kit (Thermo Fisher Scientific, Inc.) according to the manufacturer's instructions.

Silencing of hTERT by RNA interference. The cells (FB-TERT fibroblasts) were plated prior to transfection for a period of $24 \mathrm{~h}$. They were then transfected with hTERT siRNA using $\mathrm{X}$-treme gene siRNA reagent (Roche Diagnostics, Basel, Switzerland). The siRNAs were synthesized by Shanghai GenePharma Co., Ltd. (Zhangjiang Hi-Tech Park, Shanghai). The siRNA sequences were as follows: hTERT siRNA sense, 5'-GGAAGAGUGUCUGGAGCAATT-3' and antisense, 5'-UUGCUCCAGACACUCUUCCTT-3'. The negative control (scramble) siRNA sequence was as follows: sense, 5'-UUCU CCGAACGUGUCACGUTT-3' and antisense, 5'-ACGUGA CACGUUCGGAGAATT-3'.

Telomerase activity assay. Telomerase activity was measured using the telomerase repeat amplification protocol enzyme-linked immunosorbent assay (ELISA). The PCR-ELISA kit was provided by Roche Diagnostics GmbH (Mannheim, Germany). The PCR product was detected with an antibody against digoxigenin that was conjugated to peroxidase. The probe was visualized by virtue of peroxidase-metabolizing TMB color liquid to form a colored reaction product, and the absorbance of the samples was measured at $450 \mathrm{~nm}$ using an ELISA microtiter reader (Epoch; BioTek Instruments, Winooski, VT, USA).

Detection of autophagy. The cells were digested and washed twice with PBS (pH 7.4; HyClone, Logan, UT, USA), centrifuged for $5 \mathrm{~min}$ at $37^{\circ} \mathrm{C}$, and resuspended in a binding buffer. Autophagosomes were marked using the Cyto-ID Autophagy Detection kit (Enzo Life Sciences, Inc., Farmingdale, NY, USA). Marked cells were photographed under a fluorescence microscope (Nikon), and the fluorescence intensity was detected using a flow cytometer (FC500 MPL; Beckman Coulter, Inc., Brea, CA, USA).

Detection of apoptosis. The digested cells were washed twice with PBS (pH 7.4; HyClone), centrifuged for $5 \mathrm{~min}$ at $37^{\circ} \mathrm{C}$, and resuspended in binding buffer. They were then stained using the Annexin V-FITC Apoptosis Detection kit, followed by the addition of propidium iodide (PI) (both from BD Biosciences, San Jose, CA, USA). The samples were then analyzed by flow cytometry.

ROS generation assay. Intracellular ROS production was measured using 2',7'-dichlorodihydrofluorescein diacetate (DCFH-DA) (Beyotime Institute of Biotechnology, Shanghai, China). The fibroblasts were seeded in 60-mm culture dishes. The cells were then subjected to hypoxic conditions for 24 and $48 \mathrm{~h}$ and were then incubated with DCFH-DA (20 mM) for $20 \mathrm{~min}$ at $37^{\circ} \mathrm{C}$ in a dark place. Following incubation, the cells were harvested using trypsin-EDTA solution. The cell suspensions were then centrifuged for $5 \mathrm{~min}$ at room temperature and the supernatant was removed. The fluorescence intensity of DCFH-DA was measured and calculated using a flow cytometer (FC500 MPL; Beckman Coulter, Inc.).

Measurement of mitochondrial membrane potential $(\Delta \Psi \mathrm{m})$. Loss of $\Delta \Psi \mathrm{m}$ was assessed by flow cytometry with 5,5',6,6'-tetrachloro-1,1',3,3'-tetraethylbenzimidazole-carbocyanide iodine (mitochondrial membrane potential assay kit with JC-1; Beyotime Institute of Biotechnology) staining. The cells were collected into $1.5 \mathrm{ml}$ tubes and incubated with JC-1 for $20 \mathrm{~min}$ at $37^{\circ} \mathrm{C}$. The fluorescence was detected at excitation and emission wavelengths of 525 and $590 \mathrm{~nm}$, respectively. Red emission from the dye is attributed to a potential-dependent aggregation of JC-1 in the mitochondrial. Green fluorescence reflects the monomeric form of JC-1, appearing in the cytoplasm after mitochondrial membrane depolarization. The $\Delta \Psi \mathrm{m}$ of the cells in each treatment group was calculated as the fluorescence ratio of red to green. Carbonylcyanide-m-chlorop henylhydrazone (CCCP) is a protonophore, which can cause the dissipation of $\Delta \Psi \mathrm{m}$ and was used as a positive control.

Assessment of intracellular glutathione to glutathione disulfide (GSH/GSSG) levels. The cells were seeded for $24 \mathrm{~h}$ in a 6 -well plate prior to transfection at $37^{\circ} \mathrm{C}$. Following transfection under hypoxic or normoxic conditions $\left(21 \% \mathrm{O}_{2}\right)$, the cells were washed once with chilled PBS. The GSH assay was optimized from the protocol of the GSH and GSSG assay kit (Beyotime Institute of Biotechnology).

Statistical analysis. All experiments were performed at least 3 times for statistical significance. Numerical data are expressed as the means \pm SD. Statistical analysis was performed using the paired Student t-test. A value of $\mathrm{P}<0.05$ was considered to indicate a statistically significant difference.

\section{Results}

Histological changes in rectal tissue following radiation. The histological changes following irradiation were characterized by the loss of the surface epithelium, deep erosion of the mucosa, thickening of the lamina propria and submucosa (Fig. 1A and B), collagen deposition and fibrosis (Fig. 1C and D). The severity of the damage increased with time. In the unirradiated control rectal tissue, no such lesions were observed.

HIF-1 $\alpha$ and TERT protein levels in irradiated rectal tissue. The HIF-1 $\alpha$ and TERT protein levels in the irradiated rectal 

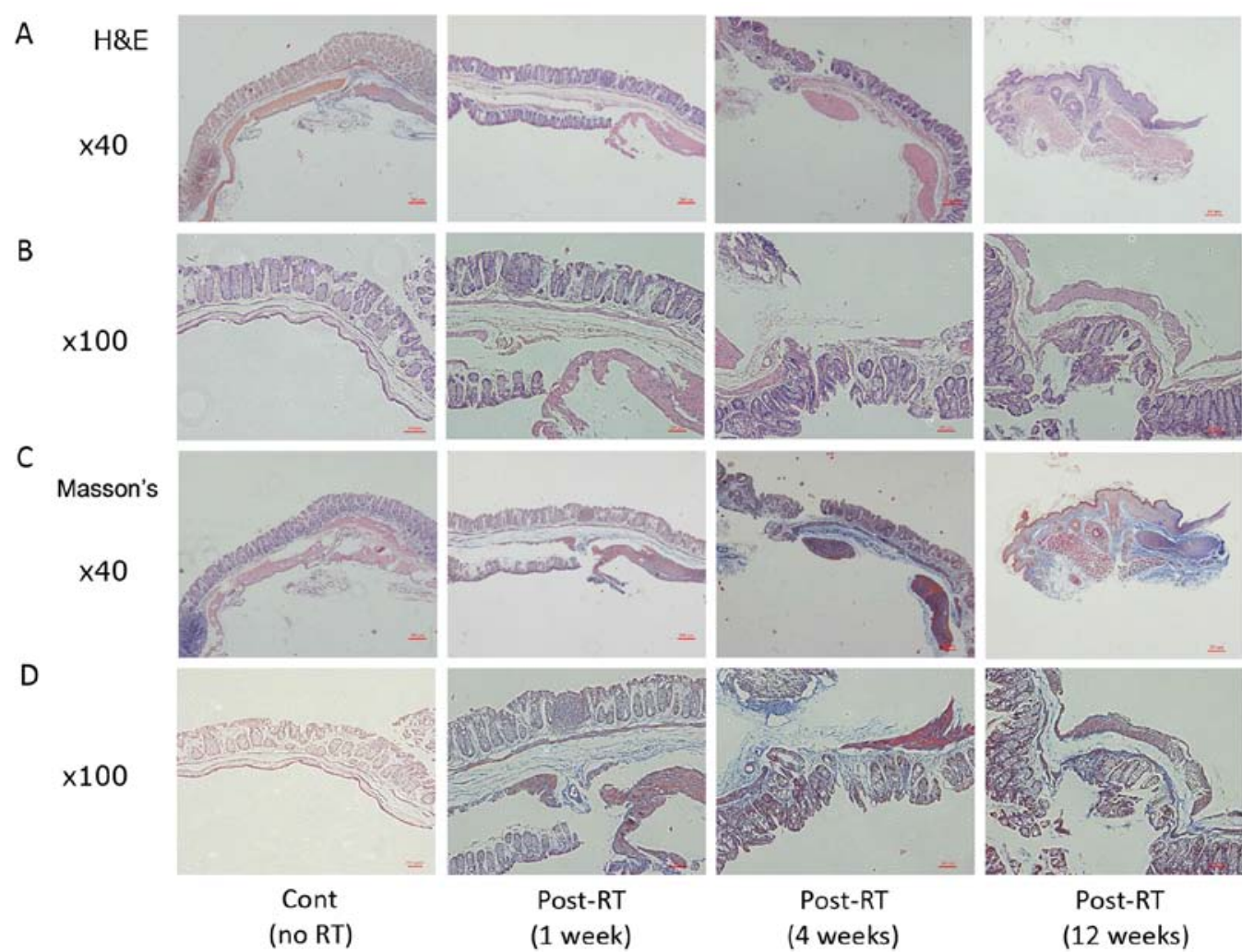

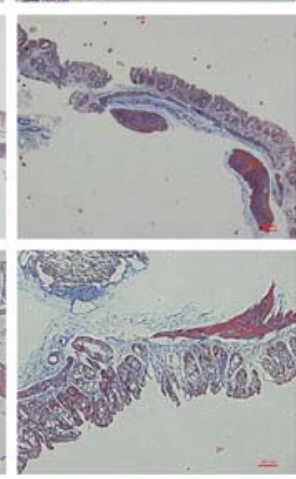

Post-RT

(4 weeks)

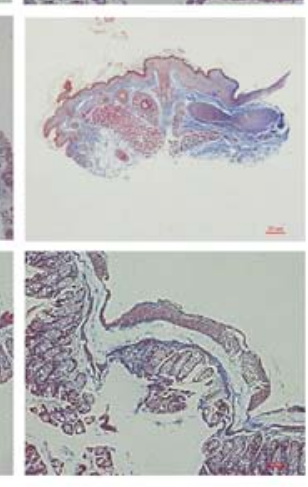

Post-RT

(12 weeks)

Figure 1. Representative histological changes in rectal tissue following irradiation. (A and B) Hematoxylin and eosin (H\&E) staining was performed for the morphological lesions and (C and D) Masson's staining was performed for collagen deposition. Blue staining indicates fibrosis. Cont, unirradiated control mice; RT, radiation treatment at a dose of 25 Gy; post-RT: 1, 4 and 12 weeks post-irradiation. Magnification: (A and C) x40; (B and D) x100. Scale bars: (A and C) 2 $00 \mu \mathrm{m}$ and (B and D) $100 \mu \mathrm{m}$.

A HIF-1 $1 \alpha$ $\times 200$

B

C

TERT

$\times 400$
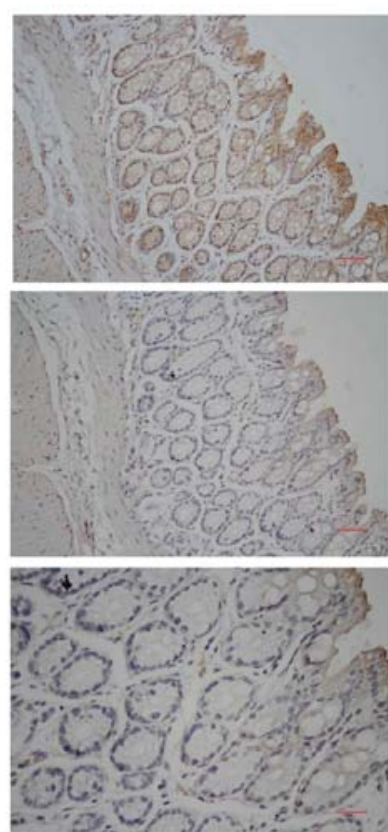

Cont

(no RT)
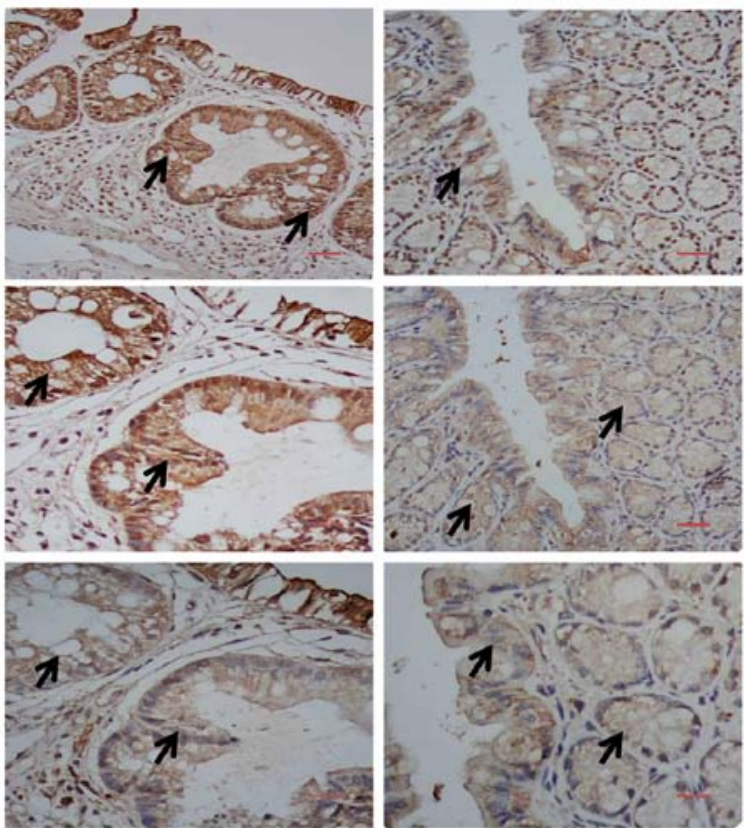

Post-RT

(4 weeks)

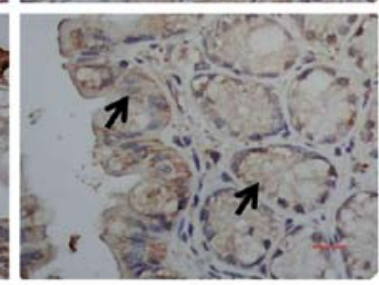

Post-RT

(12 weeks)

Figure 2. Immunohistochemical staining of irradiated rectal tissue. (A) Hypoxia inducible factor-1 $\alpha$ (HIF-1 $\alpha$ ), (B and C) telomerase reverse transcriptase (TERT), Brown staining indicates positive staining. Cont, unirradiated control mice; RT, radiation treatment at a dose of 25 Gy; post-RT, 4 and 12 weeks post-irradiation. Magnification: (A and B) x200; (C) x400. Scale bars: (A and B) $50 \mu \mathrm{m}$ and (C) $25 \mu \mathrm{m}$. Arrows indicate HIF-1 $\alpha$ or hTERT protein expression.

tissue were analyzed. As shown in Fig. 2, the HIF-1 $\alpha$ and TERT protein levels increased significantly 4 and 12 weeks post-irradiation. TERT protein translocated from the nucleus to the cytoplasm following irradiation, which was more evident 
in the 12 weeks post-RT group (Fig. 2C). Brown staining indicated positive staining and increased expression in the irradiated tissues.

Effect of TERT on apoptosis and ROS production under hypoxic conditions. The TERT-overexpressing fibroblasts were successfully generated. The FB-TERT fibroblasts were smaller (Fig. 3A) than the original fibroblasts (FBs). To assess the efficiency of transfection, the relative mRNA expression of hTERT was determined in the FBs and FB-TERT fibroblasts; hTERT mRNA expression was significantly higher in the FB-TERT fibroblasts (Fig. 3A). The fibroblasts transfected with TERT siRNA (TERTi) exhibited a decreased TERT mRNA expression and telomerase activity (Fig. 3B and C). To determine whether TERT affects apoptosis under hypoxic conditions, we examined the apoptosis level following different durations of exposure to hypoxia. As shown in Fig. 3D, exposure to hypoxic conditions (low oxygen concentrations) induced cell apoptosis in a time-dependent manner compared to the cells exposed to normoxic conditions. The TERT-overexpressing fibroblasts exhibited a significantly reduced percentage of apoptotic cells under normoxic and hypoxic conditions compared to the FBs transfected with the empty vector and to those transfected with hTERT siRNA (Fig. 3D). The TERT-overexpressing fibroblasts transfected with the negative control siRNA exhibited a significant reduction in ROS production, as compared to the cells transfected with hTERT siRNA (Fig. 3E).

Silencing of TERT reduces autophagy levels in fibroblasts. The activation of autophagy was analyzed on the basis of the fluorescence intensity of autophagosomes (Fig. 4A) and the expression of LC-3 in fibroblasts (Fig. 4B). The fibroblasts transfected with hTERT siRNA exhibited reduced autophagy levels under hypoxic conditions. Similarly, they also had a lower ratio of LC3-II to LC3-I expression than that observed in the FB-TERT fibroblasts transfected with the negative control siRNA. To evaluate the association between autophagy, ROS and apoptosis, the autophagy inhibitor, 3-MA, and a ROS scavenger were used. As shown in Fig. 4C, the fibroblasts treated with the autophagy inhibitor exhibited increased ROS production under hypoxic conditions, which indicates that autophagy leads to the scavenging of redundant ROS. The fibroblasts treated with the ROS scavenger exhibited moderate apoptosis. However, the inhibition of autophagy markedly increased apoptosis, which indicates a protective effect of autophagy against cell apoptosis by ROS scavenging (Fig. 4D).

TERT promotes autophagy through $p-N F-\kappa B$ activation. The fact that TERT promotes autophagy was demonstrated (Fig. 4A and $\mathrm{B}$ ), and thus $\mathrm{NF}-\kappa \mathrm{B}$ was examined as a medium between TERT and autophagy. p-NF- $\mathrm{B}$ p65 expression was assessed in the FB-TERT fibroblasts transfected with hTERT siRNA or negative control siRNA. An evident reduction in p-NF- $\mathrm{kB}$ p65 expression was observed in the fibroblasts in which hTERT expression was silenced (Fig. 5A). In addition, in the TERToverexpressing fibroblasts, $\mathrm{p}-\mathrm{NF}-\kappa \mathrm{B}$ p65 was activated and translocated to the nucleus, which could be significantly assayed, while no obvious changes were observed in the cytoplasm (Fig. 5B). At the same time, the inhibition of p-NF- $\kappa$ B p65 activation by BAY11-7082 reduced the ratio of
LC3-II to LC3-I expression compared to that observed in the dimethyl sulphoxide (DMSO)-treated group (Fig. 5C), which indicates that TERT promotes autophagy through the activation of NF-kB.

TERT-overexpressing fibroblasts exhibit an enhanced GSH antioxidant defense capacity and a more stable $\Delta \Psi m$ under hypoxic conditions. To determine whether TERT affects the cell antioxidant defense capacity, the ratio of GSH/GSSG was assessed, which alters cellular capacity in order to resist oxidative stress. As such, fibroblasts overexpressing TERT or or the FB-TERT fibroblasts transfected with hTERT siRNA were exposed to normoxic or hypoxic conditions for $24 \mathrm{~h}$. Exposure to hypoxia reduced the ratio of GSH/GSSG, and the fibroblasts transfected with hTERT siRNA maintained a lower ratio of GSH/GSSG (Fig. 6A), which provides evidence indicating that TERT exerts protective effects on GSH metabolism and against oxidative insults.

Since the inhibitory effect of TERT on intracellular ROS production and its protective antioxidant effects had been established, we then assessed $\Delta \Psi \mathrm{m}$, which provides information about mitochondrial membrane permeabilization. It has been shown that hypoxic conditions lead to a decrease in $\Delta \Psi \mathrm{m}$. The decrease in $\Delta \Psi \mathrm{m}$ in the cells transfected with hTERT siRNA was greater than that observed in the TERT-overexpressing cells (Fig. 6B), which provides further evidence indicating that TERT expression endows cells with a survival advantage against ROS production induced by hypoxia by inhibiting mitochondrial permeabilization.

\section{Discussion}

Radiation-induced late injury to normal tissue surrounding the tumor is a significant dose-limiting factor of radiation therapy. The pathogenesis of radiation proctitis has not yet been completed elucidated; it begins with initial radiation-induced mucosal injury, which is followed by collagen deposition in the ECM, tissue remodeling and fibrosis, and subsequently, the tissue response to ischemia (20). Previous studies have demonstrated that hypoxia following irradiation is an inevitable injury mechanism that is related to changes in the levels of several cytokines, such as HIF-1 $\alpha$, VEGF, TGF- $\beta$ and CD31. The overexpression of TGF- $\beta$ induced by irradiation leads to the accumulation of pathological amounts of the ECM in rectal tissues (4) and lung tissues $(21,22)$. Hypoxia can induce oxidative damage, mainly caused by ROS, which induces cell death through mitochondrial dysfunction $(23,24)$.

TERT is essential to the maintenance of telomerase, which contributes to cell proliferation $(12,13)$. Hypoxia not only transactivates hTERT promoter activity, but also enhances endogenous hTERT expression. The introduction of an antisense oligonucleotide for HIF-1 $\alpha$ diminishes hTERT expression during hypoxia, indicating that the upregulation of hTERT by hypoxia is directly mediated through HIF-1 $\alpha$ (18). In the present study, we demonstrate that increased TERT expression significantly antagonizes the increase in cellular ROS in response to hypoxia. GSH loss and oxidation are caused by the increased expression of the rate-limiting enzyme of GSH synthesis, that is, GCLC. Studies have proven that an early and sustained induction of GCLC occurs in response to oxidative stress in 

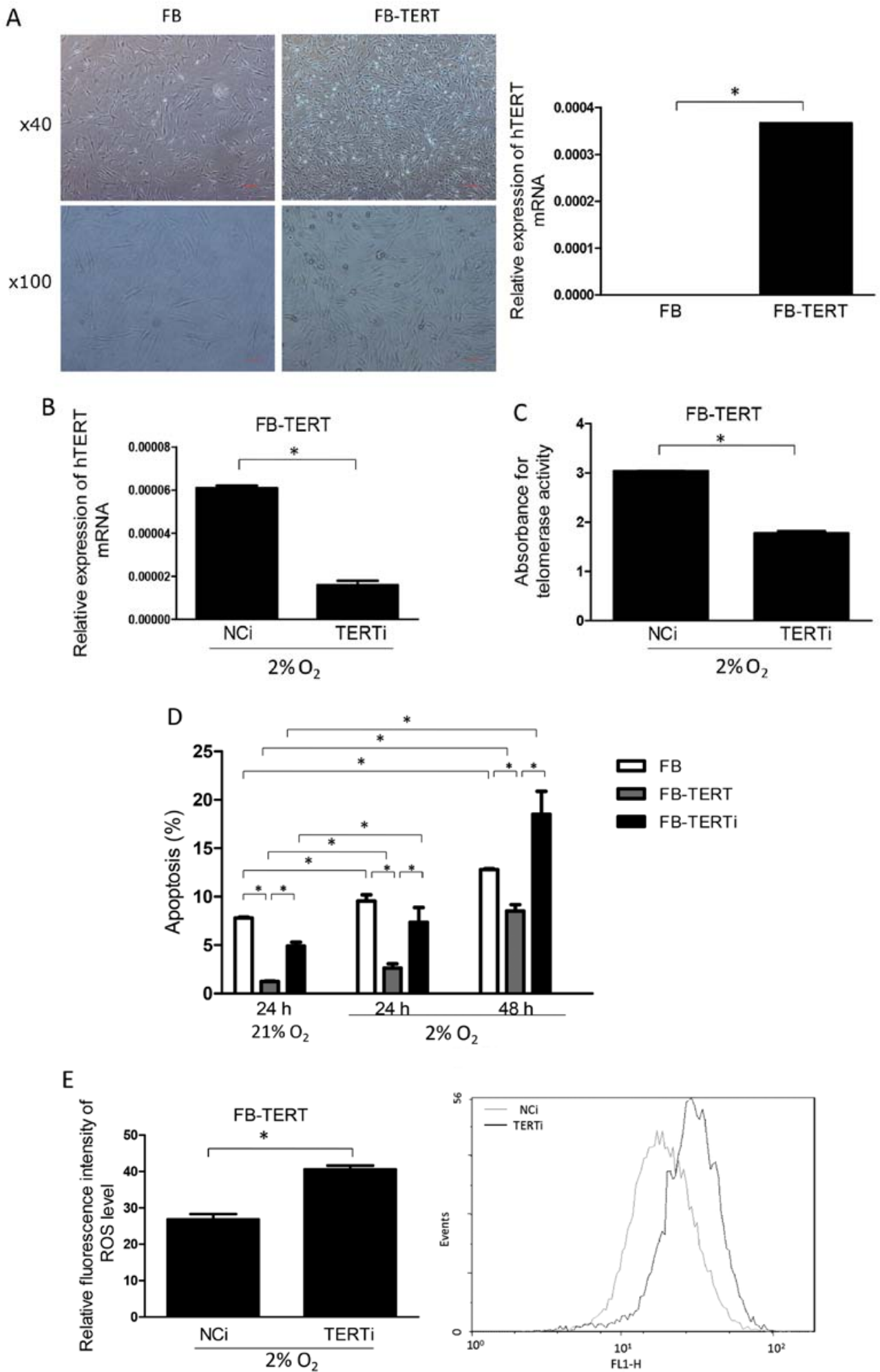

Figure 3. Generation of fibroblasts overexpressing human telomerase reverse transcriptase (hTERT), and the effects of TERT on hypoxia-induced apoptosis and reactive oxygen species (ROS) production in human fibroblast cells. hTERT- overexpressing fibroblasts were generated via lentiviral transduction. (A) Cell morphological changes following the overexpression of hTERT and the relative mRNA expression of hTERT in FB and FB-TERT fibroblasts. (B and C) The expression of TERT mRNA and telomerase activity level following transfection of FB-TERT cells with TERT siRNA under hypoxic conditions ( $\left.2 \% \mathrm{O}_{2}, 24 \mathrm{~h}\right)$. (D) The percentage of apoptotic cells under different oxygen concentrations and exposure times. (E) Cellular ROS production under hypoxic conditions was assessed using the probe 2',7'-dichlorodihydrofluorescein diacetate (DCFH-DA) following transfection of in FB-TERT cells with TERT siRNA. Three independent images per point were analyzed. FB-TERT, hTERT-overexpressing cells. (A) Magnification, $x 40$ and x100. (A and C) Scale bars, $200 \mu \mathrm{m} ;$ FB-TERTi and TERTi, TERT siRNA-transfected group. NCi, negative control for scramble siRNA-transfected group of FB-TERT cells. Data are the means \pm SD of 3 experiments, ${ }^{*} \mathrm{P}<0.05$.

hTERT-overexpressing cells $(19,25)$, which helps to antagonize a rapid decline in cellular GSH levels, and maintaining a higher
GSH/GSSG level. Furthermore, the partial rescue from the decrease in $\Delta \Psi \mathrm{m}$ in the TERT-overexpressing group provided 
A
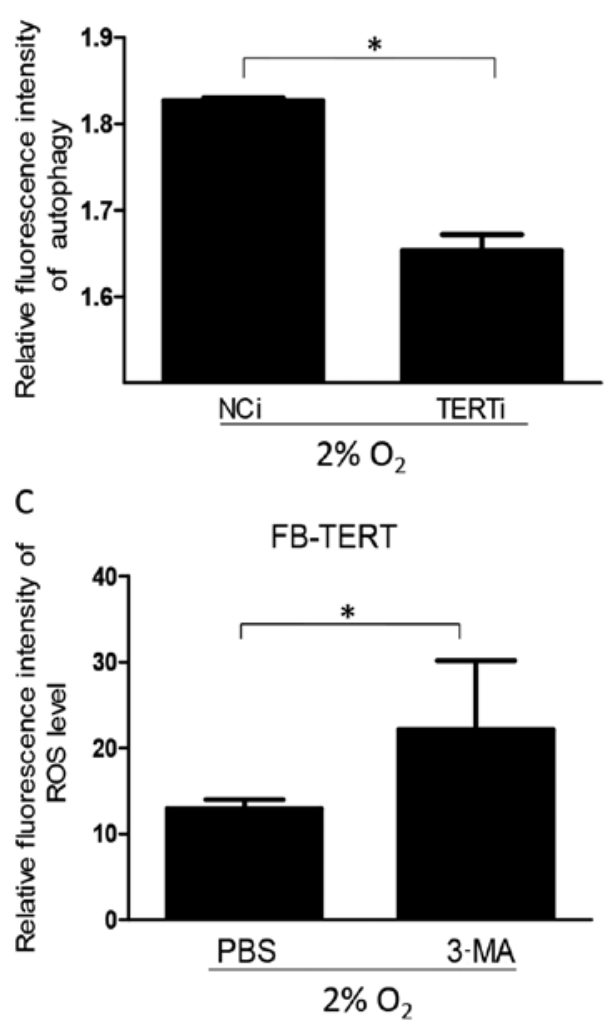

B

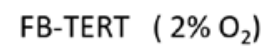

LC3-I

LC3-II

LC3-II/I

GAPDH

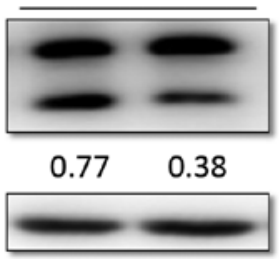

$\mathrm{NCi}$ TERTi

D

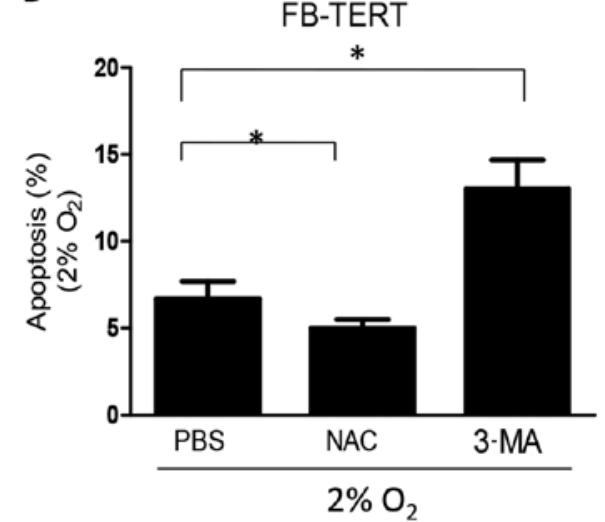

Figure 4. Effects of telomerase reverse transcriptase (TERT) on autophagy levels in human fibroblasts and the role of cell autophagy in reactive oxygen species (ROS) production and apoptosis under hypoxic conditions. (A) The level of autophagosome formation was measured quantitatively using a fluorescence intensity assay of FB-TERT cells. (B) Expression of LC3-II and LC3-I was assessed by western blot analysis of FB-TERT cells. (C and D) Treatment with the autophagy inhibitor, 3-methyladenine (3-MA) (at the concentration of $5 \mu \mathrm{M}$ ), promoted the production of ROS and led to apoptosis, and ROS scavenging with NAC (at the concentration of $3 \mu \mathrm{M}$ ) decreased the percentage of apoptotic cells relative to that for phosphate-buffered saline (PBS) treatment. TERTi, TERT siRNA-transfected group of FB-TERT cells. NCi, negative control for scramble siRNA-transfected group of FB-TERT cells. 3-MA, 3-MA treatment group; NAC, $N$-acetylcysteine treatment group; PBS, PBS treatment group as a negative control. Data are the means \pm SD of 3 experiments, ${ }^{*} \mathrm{P}<0.05$
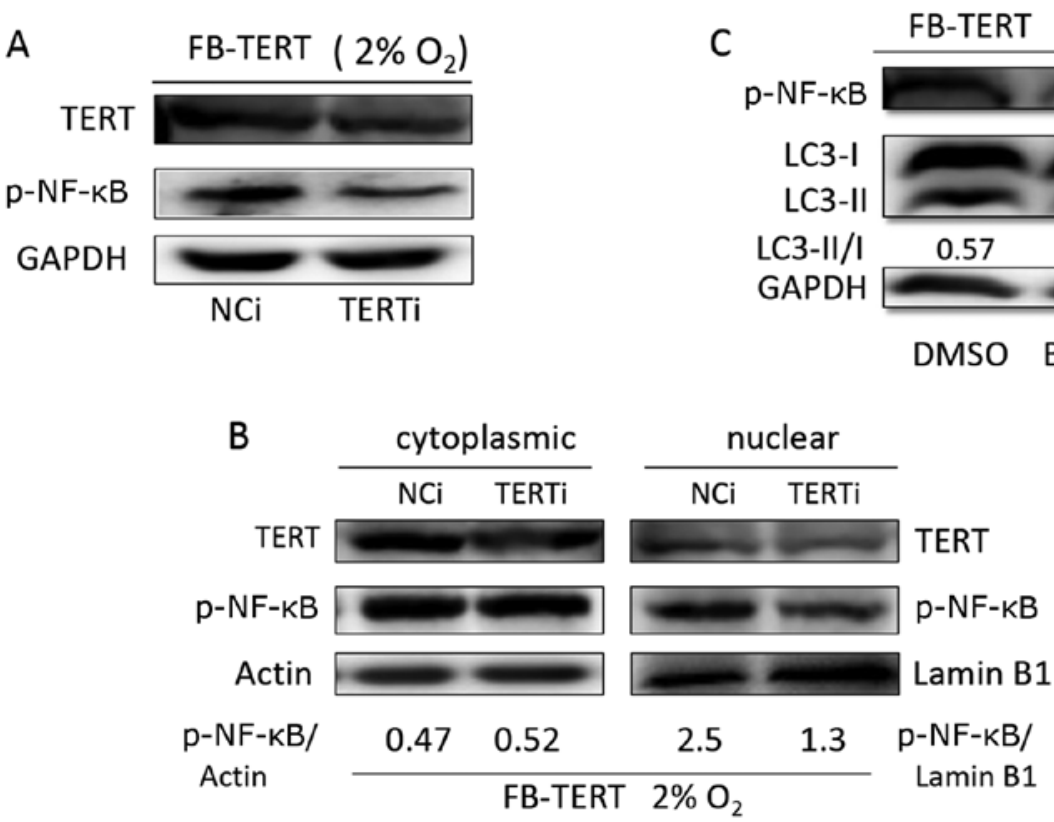

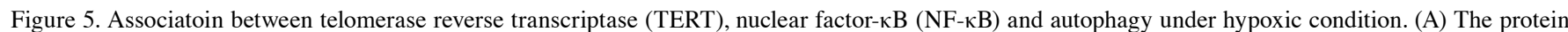
expression of TERT and p-NF- $\mathrm{KB}$ p65 following transfection with TERT siRNA. (B) Levels of autophagy-related proteins, LC3-II and LC3-I, after thye inhibition of p-NF- $\mathrm{kB}$ p65 by treatment with BAY11-7082 at the concentration of $10 \mu \mathrm{M}$ and dimethyl sulphoxide (DMSO) treatment. (C) The expression of p-NF-kB p65 after the inhibition of TERT in the cytoplasm and nucleus, respectively, under hypoxic conditions. TERTi, TERT siRNA-transfected group; NCi, negative control for scramble siRNA-transfected group; BAY11-7082, p-NF-kB inhibitor; DMSO, DMSO treatment group as a negative control. 

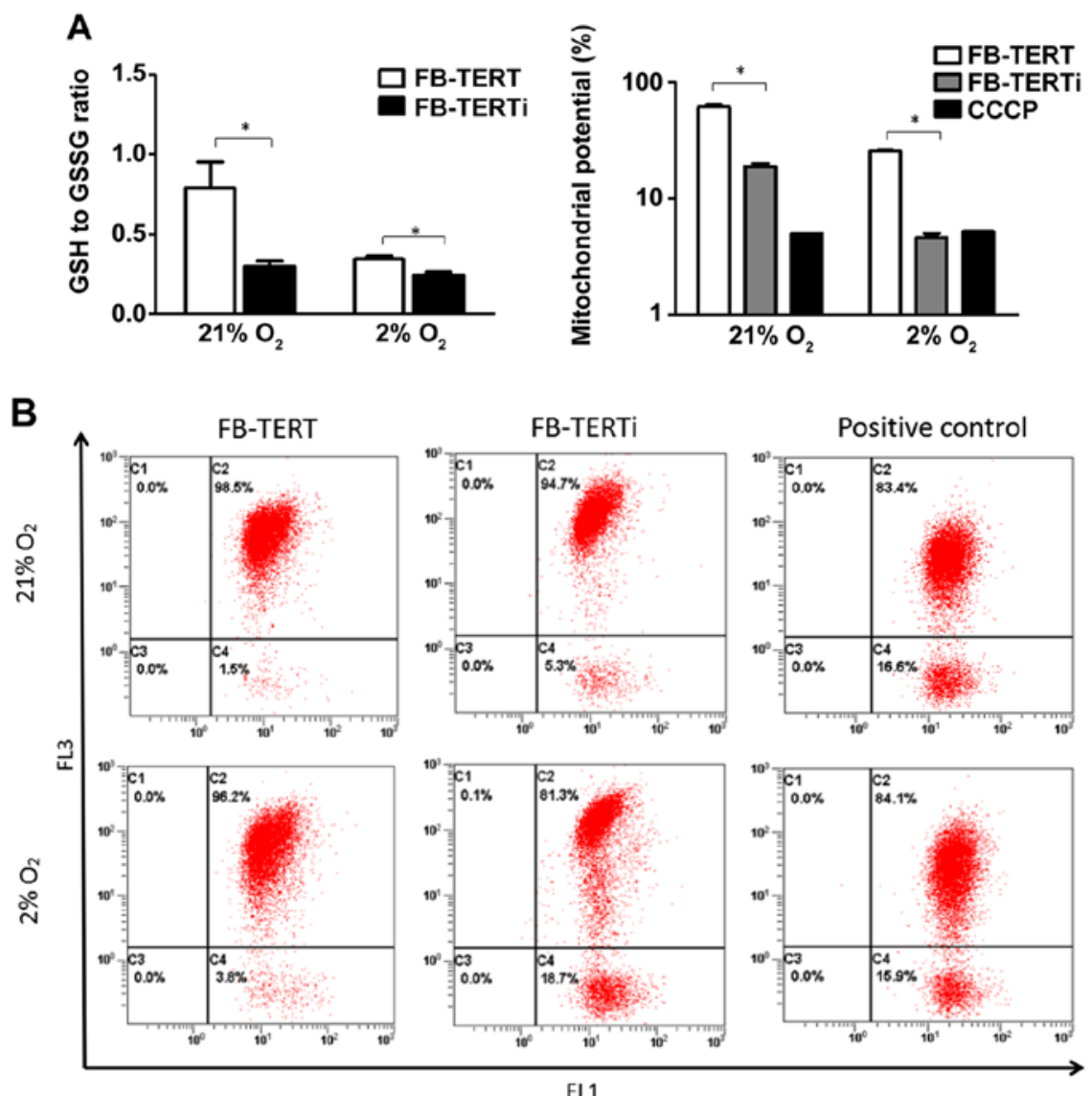

Figure 6. Cells transfected with telomerase reverse transcriptase (TERT) siRNA exhibited a reduction in glutathione (GSH) antioxidant defense and mitochondrial membrane potential. (A) Intracellular ratio of GSH to GSH disulfide (GSH/GSSG) was computed under normoxic and hypoxic conditions. (B) The fluorescence value of red and green was determined by flow cytometry, after staining with JC-1. The ratio of red/green fluorescent represents the mitochondrial membrane potential. FB-TERTi, TERT siRNA-transfected group; carbonylcyanide-m-chlorophenylhydrazone (CCCP), CCCP treatment as a positive group. Data are the means $\pm \mathrm{SD}$ of 3 experiments, ${ }^{*} \mathrm{P}<0.05$.

further evidence that TERT endows cells with a survival advantage against ROS-mediated cell toxic effects by inhibiting mitochondrial permeabilization. In addition to antagonizing cellular ROS, the activation of autophagy under hypoxic conditions was demonstraed in our study as an underlying mechanism of the reducing effects of TERT on the production of ROS.

Hypoxia is an inducer of autophagy, which is a lysosom-mediated degradation process for non-essential or damaged cellular constituents and serves to preserve the balance between organelle biogenesis, protein synthesis and clearance (26). The association between autophagy and cell death is complex, as autophagy can be involved in either cell death or survival, depending on the cellular context $(27,28)$. Previous studies have demonstrated that autophagy can protect cells from radio- and chemotherapy in breast, pharyngeal, cervical, lung and rectal tumors (29-31). Another study demonstrated that hypoxia-induced autophagy is also involved in a HIF-1 $\alpha$-mediated general mechanism of cell survival. BNIP3 and BNIP3L are downstream targets of HIF-1 $\alpha$-mediated autophagy (24). The inhibition of autophagy by the addition of chloroquine or the prevention of K63 ubiquitination increases the formation of ROS (32). Our study demonstrates that autophagy acts as a survival mechanism in cells exposed to hypoxia through ROS reduction, which is in agreement with the findings of these previous studies.
To investigate the mediator between TERT and autophagy, we hypothesized that $\mathrm{NF}-\kappa \mathrm{B}$ may be a possible candidate. The activation of the canonical NF- $\kappa \mathrm{B}$ pathway is closely associated with the regulation of autophagy $(33,34) . N F-\kappa B$, a transcription factor, which plays an important role in the response to cell damage, stress and inflammatory reactions, has been reported to have a unique crosstalk with ROS (35). The mammalian NF- $\mathrm{B}$ family consists of 5 subunits: RelA/ p65, RelB, c-Rel, p50 and p52, and NF- $\kappa$ B is a heterodimer composed of p65 and p50. Under normal conditions, NF- $\kappa \mathrm{B}$ exists in the cytoplasm of the cell in an inactive state through interactions with the inhibitor of $\mathrm{NF}-\kappa \mathrm{B}$. When exposed to specific stimuli, NF- $\kappa \mathrm{B}$ is activated and is rapidly transferred to inside the nucleus where it is responsible for controlling transcription (36). Studies have also reported NF- $\kappa \mathrm{B}$ may be a target of hTERT. Indran et al demonstrated higher levels of NF- $\mathrm{B}$ p65 in hTERT-overexpressing cells, which also exhibited a significantly higher level of manganese-dependent superoxide dismutase (MnSOD) (19). In this study, we uncovered a reduction of p-NF- $\mathrm{B}$ p65 transfer into the nucleus with a decrease in TERT expression, indicating an activating effect of TERT on NF- $\mathrm{B}$, which is is accordance with these studies.

Taken together, our present study demonstrated that hypoxia and TERT expression increased in rectal fibrotic tissues following irradiation. Further experiments revealed that 
hypoxia induced cell apoptosis by promoting ROS production TERT promoted autophagy by activating $\mathrm{p}-\mathrm{NF}-\kappa \mathrm{B}$, which enabled the modulation of intracellular ROS under hypoxic conditions by maintaining the antioxidant status and mitochondrial function, and blunting apoptotic signals. In the future, studies on the effects of hypoxia and TERT in animal models are warranted. TERT may prove to be target with which to enhance the anti-apoptotic ability of cells by enhancing the cellular autophagy level, which may be beneficial for the reduction of radiation-induced injury to normal tissue.

\section{Acknowledgements}

This study was supported by the Scientific Research Foundation for the Returned Overseas Chinese Scholars (no. N130204) from the China State Education Ministry, the National Natural Science Foundation of China (nos. 81202148 and 31370838), the Shanghai Pujiang Program (no. 13P1401600), and the Foundation of Shanghai Committee of Science and Technology of China (no. 12DZ2260100).

\section{References}

1. Grodsky MB and Sidani SM: Radiation proctopathy. Clin Colon Rectal Surg 28: 103-111, 2015.

2. Christofidou-Solomidou M, Pietrofesa RA, Arguiri E, Schweitzer KS, Berdyshev EV, McCarthy M, Corbitt A, Alwood JS, Yu Y, Globus RK, et al: Space radiation-associated lung injury in a murine model. Am J Physiol Lung Cell Mol Physiol 308: L416-L428, 2015.

3. Nordal RA, Nagy A, Pintilie M and Wong CS: Hypoxia and hypoxia-inducible factor-1 target genes in central nervous system radiation injury: a role for vascular endothelial growth factor. Clin Cancer Res 10: 3342-3353, 2004.

4. Liu Y, Kudo K, Abe Y, Aoki M, Hu DL, Kijima H and Nakane A: Hypoxia expression in radiation-induced late rectal injury. J Radiat Res (Tokyo) 49: 261-268, 2008.

5. Liu Y, Kudo K, Abe Y, Hu DL, Kijima H, Nakane A and Ono K: Inhibition of transforming growth factor-beta, hypoxia-inducible factor-1alpha and vascular endothelial growth factor reduced late rectal injury induced by irradiation. J Radiat Res (Tokyo) 50: 233-239, 2009.

6. Distler JH, Jüngel A, Pileckyte M, Zwerina J, Michel BA, Gay RE, Kowal-Bielecka O, Matucci-Cerinic M, Schett G, Marti HH, et al: Hypoxia-induced increase in the production of extracellular matrix proteins in systemic sclerosis. Arthritis Rheum 56: 4203-4215, 2007.

7. Haroon ZA, Raleigh JA, Greenberg CS and Dewhirst MW: Early wound healing exhibits cytokine surge without evidence of hypoxia. Ann Surg 231: 137-147, 2000.

8. Schmidt-Ullrich RK, Dent P, Grant S, Mikkelsen RB and Valerie K: Signal transduction and cellular radiation responses. Radiat Res 153: 245-257, 2000.

9. Spilsbury A, Miwa S, Attems J and Saretzki G: The role of telomerase protein TERT in Alzheimer's disease and in tau-related pathology in vitro. J Neurosci 35: 1659-1674, 2015.

10. Greider CW: Chromosome first aid. Cell 67: 645-647, 1991

11. De Boeck G, Forsyth RG, Praet M and Hogendoorn PC: Telomere-associated proteins: cross-talk between telomere maintenance and telomere-lengthening mechanisms. J Pathol 217: 327-344, 2009

12. Ahmed S, Passos JF, Birket MJ, Beckmann T, Brings S, Peters H, Birch-Machin MA, von Zglinicki T and Saretzki G: Telomerase does not counteract telomere shortening but protects mitochondrial function under oxidative stress. J Cell Sci 121: 1046-1053, 2008.

13. Masutomi K, Possemato R, Wong JM, Currier JL, Tothova Z, Manola JB, Ganesan S, Lansdorp PM, Collins K and Hahn WC: The telomerase reverse transcriptase regulates chromatin state and DNA damage responses. Proc Natl Acad Sci USA 102 8222-8227, 2005.
14. Jan HM, Wei MF, Peng CL, Lin SJ, Lai PS and Shieh MJ: The use of polyethylenimine-DNA to topically deliver hTERT to promote hair growth. Gene Ther 19: 86-93, 2012.

15. Qu Y, Duan Z, Zhao F, Wei D, Zhang J, Tang B, Li J, Yang C and Mu D: Telomerase reverse transcriptase upregulation attenuates astrocyte proliferation and promotes neuronal survival in the hypoxic-ischemic rat brain. Stroke 42: 3542-3550, 2011.

16. Tao X, Ming-Kun Y, Wei-Bin S, Hai-Long G, Rui K and Lai-Yong T: Role of telomerase reverse transcriptase in glial scar formation after spinal cord injury in rats. Neurochem Res 38: 1914-1920, 2013.

17. Osanai M, Tamaki T, Yonekawa M, Kawamura A and Sawada N: Transient increase in telomerase activity of proliferating fibroblasts and endothelial cells in granulation tissue of the human skin. Wound Repair Regen 10: 59-66, 2002.

18. Nishi H, Nakada T, Kyo S, Inoue M, Shay JW and Isaka K: Hypoxia-inducible factor 1 mediates upregulation of telomerase (hTERT). Mol Cell Biol 24: 6076-6083, 2004.

19. Indran IR, Hande MP and Pervaiz S: hTERT overexpression alleviates intracellular ROS production, improves mitochondrial function, and inhibits ROS-mediated apoptosis in cancer cells. Cancer Res 71: 266-276, 2011.

20. Okunieff P, Cornelison T, Mester M, Liu W, Ding I, Chen Y, Zhang H, Williams JP and Finkelstein J: Mechanism and modification of gastrointestinal soft tissue response to radiation: role of growth factors. Int J Radiat Oncol Biol Phys 62: 273-278, 2005.

21. Yang S, Zhang M, Chen C, Cao Y, Tian Y, Guo Y, Zhang B, Wang X, Zhang L et al: Triptolide mitigates radiation-induced pulmonary fibrosis. Radiat Res 184: 509-517, 2015.

22. Martin M, Lefaix J and Delanian S: TGF-betal and radiation fibrosis: a master switch and a specific therapeutic target? Int J Radiat Oncol Biol Phys 47: 277-290, 2000.

23. Murphy MP: How mitochondria produce reactive oxygen species. Biochem J 417: 1-13, 2009.

24. Lee J, Giordano S and Zhang J: Autophagy, mitochondria and oxidative stress: cross-talk and redox signalling. Biochem J 441: 523-540, 2012.

25. Meister A and Anderson ME: Glutathione. Annu Rev Biochem 52: 711-760, 1983.

26. Sun Y, Xing X, Liu Q, Wang Z, Xin Y, Zhang P, Hu C and Liu Y: Hypoxia-induced autophagy reduces radiosensitivity by the HIF-1 $\alpha / \mathrm{miR}-210 / \mathrm{Bcl}-2$ pathway in colon cancer cells. Int $\mathbf{J}$ Oncol 46: 750-756, 2015

27. Kroemer G and Jäättelä M: Lysosomes and autophagy in cell death control. Nat Rev Cancer 5: 886-897, 2005.

28. Baehrecke EH: Autophagy: dual roles in life and death? Nat Rev Mol Cell Biol 6: 505-510, 2005.

29. He WS, Dai XF, Jin M, Liu CW and Rent JH: Hypoxia-induced autophagy confers resistance of breast cancer cells to ionizing radiation. Oncol Res 20: 251-258, 2012.

30. Apel A, Herr I, Schwarz H, Rodemann HP and Mayer A: Blocked autophagy sensitizes resistant carcinoma cells to radiation therapy. Cancer Res 68: 1485-1494, 2008.

31. Sannigrahi MK, Singh V, Sharma R, Panda NK and Khullar M: Role of autophagy in head and neck cancer and therapeutic resistance. Oral Dis 21: 283-291, 2015.

32. Rouschop KM, Ramaekers CH, Schaaf MB, Keulers TG, Savelkouls KG, Lambin P, Koritzinsky M and Wouters BG: Autophagy is required during cycling hypoxia to lower production of reactive oxygen species. Radiother Oncol 92: 411-416, 2009.

33. Zeng M, Wei X, Wu Z, Li W, Li B, Zhen Y, Chen J, Wang P and Fei Y: NF- $\mathrm{BB}$-mediated induction of autophagy in cardiac ischemia/reperfusion injury. Biochem Biophys Res Commun 436: 180-185, 2013.

34. Zhang Y, Wu Y, Wu D, Tashiro S, Onodera S and Ikejima T: NF-kappab facilitates oridonin-induced apoptosis and autophagy in HT1080 cells through a p53-mediated pathway. Arch Biochem Biophys 489: 25-33, 2009.

35. Bubici C, Papa S, Dean K and Franzoso G: Mutual cross-talk between reactive oxygen species and nuclear factor-kappa $\mathrm{B}$ : molecular basis and biological significance. Oncogene 25: 6731-6748, 2006.

36. Sun Z, Yin Z, Liu C and Tian J: The changes in the expression of NF-KB in a degenerative human intervertebral disc model. Cell Biochem Biophys 72: 115-122, 2015. 